\title{
Tanto los intervalos intergenésicos breves como los prolongados se asocian con peores resultados perinatales
}

\author{
Both brief and prolonged birth spacing are associated with worse perinatal outcomes
}

Objetivo

Examinar la asociación entre la duración del intervalo intergenésico y el riesgo de resultados perinatales adversos.

\section{Fuente y selección de datos}

Estudios publicados en cualquier lengua. Se buscó en MEDLINE (1966-2006) EMBASE, ECLA, POPLINE, CINAHL y LILACS, actas de encuentros sobre el tema y bibliografía de los artículos encontrados. Fueron contactados investigadores - del área y autores para obtener información adicional. Fueron incluidos estudios de cohortes, de corte transversal o casoscontroles que hubieran evaluado la relación entre la duración del intervalo entre los embarazos o entre los nacimientos y cualquier resultado perinatal adverso; que hubieran ajustado sus resultados por lo menos por los dos potenciales confundidores* que los revisores juzgaron más relevantes (edad materna y nivel socioeconómico) y que hubieran usado el "intervalo entre los embarazos" como variable estratificada* en un míni-

\author{
Conde-Agudelo A, y col. JAMA. 2006;295:1809-1823
}

mo de cuatro, hubieran reportado Odds ratios ${ }^{*}$ o riesgos relativos y sus IC $95 \%$ o bien los datos individuales que permitieran calcularlos. De los 130 artículos relevantes encontrados, 67 cumplieron los criterios para ingresar a la revisión sistemática* (incluían 11.091 .959 embarazos) y 26 para la realización de la síntesis meta-análítica*

\section{Resultados principales}

Se utilizó un modelo de efecto-aleatorizado* y análisis de metaregresión ${ }^{*}$ para aunar los datos de los estudios individuales. Comparado con intervalos intergenésicos de 18 a 23 meses, los intervalos menores a seis meses se asociaron con un aumento en el riesgo de embarazo pretérmino (RNPE) bajo peso al nacer (BPN) y bajo peso para la edad gestacional (BPEG). Los intervalos entre seis y 17 meses, así como los mayores a 59 meses también se asociaron con un aumento significativo del riesgo de alguno de estos tres resultados adversos perinatales. Ver la tabla 1

Tabla 1: asociación estadística ajustada entre la duración del intervalo intergenésico y el riesgo de efectos adversos perinatales, utilizando como grupo de comparación el de las madres con intervalos de 18 a 23 meses.

\begin{tabular}{|c|c|c|c|c|c|c|}
\hline & & \multicolumn{5}{|c|}{ Odds ratio ajustadoa e IC95\% de acuerdo al intervalo intergestacional en meses } \\
\hline & & Menor a seis & Seis a 12 & 12 a 17 & 18 a 23 & Mayor a 60 \\
\hline \multicolumn{2}{|c|}{ Parto pretérmino } & $1,40(1,24$ a 1,58$)$ & $1,14(1,10$ a 1,17$)$ & $1,07(1,03$ a 0,11$)$ & 1 & $1,20(1,17$ a 1,24$)$ \\
\hline \multirow[t]{2}{*}{ Bajo peso } & al nacer & $1,61(1,39$ a 1,86$)$ & $1,14(1,10$ a 1,18$)$ & $1,05(1,01$ a 1,09$)$ & 1 & $1,43(1,27$ a 1,62$)$ \\
\hline & para la edad gestacional & $1,26(1,18$ a 1,33$)$ & $1,11(1,04$ a 1,19$)$ & $1,06(1,01$ a 1,10$)$ & 1 & $1,29(1,20$ a 1,39$)$ \\
\hline
\end{tabular}

aPor edad materna, paridad, educación, estado civil, grupo étnico, estado socio-económico, resultado perinatal previo, control prenatal, factores de riesgo médicos, estado nutricional materno, región, tabaquismo, uso de alcohol, uso de drogas, edad gestacional o peso al nacer, tipo de hospital, año del nacimiento, religión y sexo del recién nacido.

\section{Conclusiones}

Los intervalos entre los nacimientos menores a 18 meses y mayores de 59 meses se asocian significativamente con peores resultados perinatales.
Palabras clave: intervalo intergestacional, resultados perinatales, riesgo. Key words: Birth Spacing, Perinatal Outcomes, risk.

Fuente de financiamiento: Office of Population and Reproductive Health, Bureau for Global Health, US Agency for International Development.

\section{Comentario}

Si bien, ya se conocía que tanto los intervalos íntergenésicos breves como los prolongados se asocian con un aumento del riesgo de resultados perinatales adversos ${ }^{1}$; no quedaba claro si otros factores de riesgo podían actuar como confundidores ${ }^{2}$ (características maternas, nivel socioeconómico, etc.). Por ejemplo, es razonable suponer que las madres vuelven a embarazarse rápidamente son las más pobres, las de menor acceso a la educación, o a los servicios de salud, etc.

Sin embargo, para buscar respuestas a este tipo de problema sólo pueden realizarse investigaciones observacionales, en los que la mejor alternativa metodológica disponible es el análisis multivariable* con el objetivo de ajustar los resultados que pudieran estar influenciados por las dichas variables de potencial confusión. También es sabido que la calidad de una revisión sistemática y su meta-análisis depende de la calidad de los estudios que la conforman. Respecto de este trabajo, se evidenció una heterogeneidad estadística entre los diferentes estudios, lo que hace cuestionable la precisión de la medida sumaria obtenida. Sin embargo se vio una dirección de efecto similar en los resultados de todos los estudios, lo que sugeriría que el reporte del trabajo que comentamos se aproxima a la "verdad en el mundo real".

\section{Conclusiones del comentador}

El acceso a una adecuada planificación familiar con el objetivo de lograr una separación óptima de los embarazos y en consonancia con los valores de la población, podría ayudar a prevenir resultados perinatales adversos.

Gabriel Kñallevsky [ Servicio de Medicina Familiar y Comunitaria del Hospital Italiano de Buenos Aires gabriel.knallevsky@hospitalitaliano.org.ar ]

Recibido el 01/06/07 y aceptado el 23/03/08

Kñallevsky G. Tanto los intervalos intergenésicos breves como los prolongados se asocian con peores resultados perinatales. Evid Act Pract Ambul. 11(2) 43. Mar-Abr 2008. Comentado de:Conde-Agudelo A, Rosas Bermudez A, Kafury-Goeta AC. Birth Spacing and Risk of Adverse Perinatal Outcome. a Meta-analysis. JAMA. 2006;295:1809-1823.

Disponible libremente en URL: http://jama.ama-assn.org/cgi/content/full/295/15/1809\#AUTHINFO (último acceso el 23/03/08).

\section{Referencias}

1. Rousso D, y col. Effect of the interval between pregnancies on the health of mother and child. Eur J Obstet Gynecol Reprod Biol.2002;105:4-6.

2. Klebanoff MA. The interval between pregnancies and the outcome of subsequent births. N Engl J Med. 1999;340:643-644 